\title{
Kinematic analysis of Sculptor Group galaxies
}

\author{
R. H. M. Schoenmakers \\ Kapteyn Astronomical Institute, Rijksuniversiteit Groningen, The \\ Netherlands
}

\begin{abstract}
.
An analysis of the kinematics of the five major spiral galaxies in the Sculptor Group is presented. These galaxies are analyzed using the method of harmonic expansion of the velocity field as described in Schoenmakers, Franx and de Zeeuw (1997). Three different types of kinematic distortions were found: warps, lopsidedness and elongation. All the Sculptor galaxies show kinematic lopsidedness, two show signs of global elongation and two are kinematically warped. The detected kinematic distortions are well in excess of those typically found for isolated galaxies.
\end{abstract}

\section{Introduction}

The Sculptor Group is the nearest external group of galaxies at a distance of about $2.5 \mathrm{Mpc}$. Therefore, it is well suited for a careful examination of the velocity fields of the individual galaxies. Puche \& Carignan in their series of papers (see References) studied the velocity fields of the group members by means of tilted-ring modeling. In this way they deduced warp and dark matter parameters for these galaxies. However, due to non-circular motions, the rotation curves they derived may not be completely accurate. They account for this possibility by assigning larger errorbars to their derived rotation velocities. Using the method described by Schoenmakers, Franx and de Zeeuw (1997, hereafter SFdZ), we are able to assess the nature of these non-circular motions. This may teach us more about the type of non-circular motions that are likely to arise in a bound group of a few galaxies. Furthermore, we discuss possible future work on the dynamics of small galaxy groups like Sculptor. The work presented here is discussed in much more detail in the PhD-thesis of Schoenmakers, 1999, Chapter 5.

\section{Data and analysis}

\subsection{Data}

The surface density fields and velocity fields of NGC 247, NGC 55, and NGC 7793 were derived from VLA observations as presented in Braun $(1995,1997)$. The surface density fields and velocity fields of NGC 253 and NGC 300 were obtained from the NCSA Astronomy Digital Image Library (ADIL). These are the same 
fields as used in Puche, Carignan \& Bosma (1990) for NGC 300 and Puche, Carignan \& van Gorkom (1991) for NGC 253. For details regarding observations, reduction techniques and the procedures to create the fields from the data cubes, we refer to the above mentioned articles.

\subsection{Analysis}

Augmenting the standard tilted-ring procedure, Schoenmakers, Franx \& de Zeeuw (1997) presented a method to measure higher velocity harmonics present in galactic velocity fields. They show how to use these harmonics to derive fundemental parameters on the underlying gravitational well, like global elongation (using the odd velocity harmonics $m=1$ and $m=3$ ) and kinematic lopsidedness (Swaters et al. 1999). For a detailed description and derivation of the method and results for a number of field spirals, see Schoenmakers (1999).

The parameters used here to quantify non-axisymmetry in the Scultor spirals are:

- Global elongation of the potential: $\epsilon_{\text {pot }} \sin (2 \phi)$, where $\phi$ is an unknown viewing angle. Therefore, this is a statistical parameter and one must be carefull not to over-interpret low measurements. High measurements of this elongation parameter on the other hand can only represent truly highly elongated potentials.

- Kinematic lopsidedness: $\lambda_{k i n}(R)=\frac{\hat{c}_{0}(R)^{2}+\hat{s}_{2}(R)^{2}+\hat{c}_{2}(R)^{2}}{v_{\mathrm{c}}, \max ^{2}}$, the power in the "lopsidedness terms" as a fraction of the maximum rotation velocity in the galaxy.

\section{The Sculptor galaxies}

As an example of the way the harmonic method is used in quantifying the above parameters, NGC 247 will be discussed. Then, the results of the other galaxies will be given.

\subsection{Example: NGC 247}

In order to make a useful harmonic expansion, we first have to find a centre for the rings. This is done by making a tilted-ring fit (Begeman 1987) with all the parameters (inclination, position angle and centre) left free. The result of this tilted-ring fit for the position of the centre is presented in Figure 1, left panel. It seemed that the average kinematical centre was offset by about +5 " in RA and +15 " in Dec. with respect to the optical centre. But a more careful examination of the tilted-ring decompositions at various positions around the optical centre and the apparent kinematical centre led us to the conclusion that the optical centre is also the best kinematical centre. Fixing the centre at this position, a new tilted-ring fit was made for the approaching and receding sides separately. In Figure 1, right panel, we plot the rotation curves of the approaching and receding sides. The tilted-ring fits were made fixing the inclination and position angle to their average value. We see that the Southern, receding, side (indicated with triangles) has a more or less flat rotation curve, whereas the rotation curve on the Northern, approaching, side (the open circles) is flat until about 450 arcsec and 

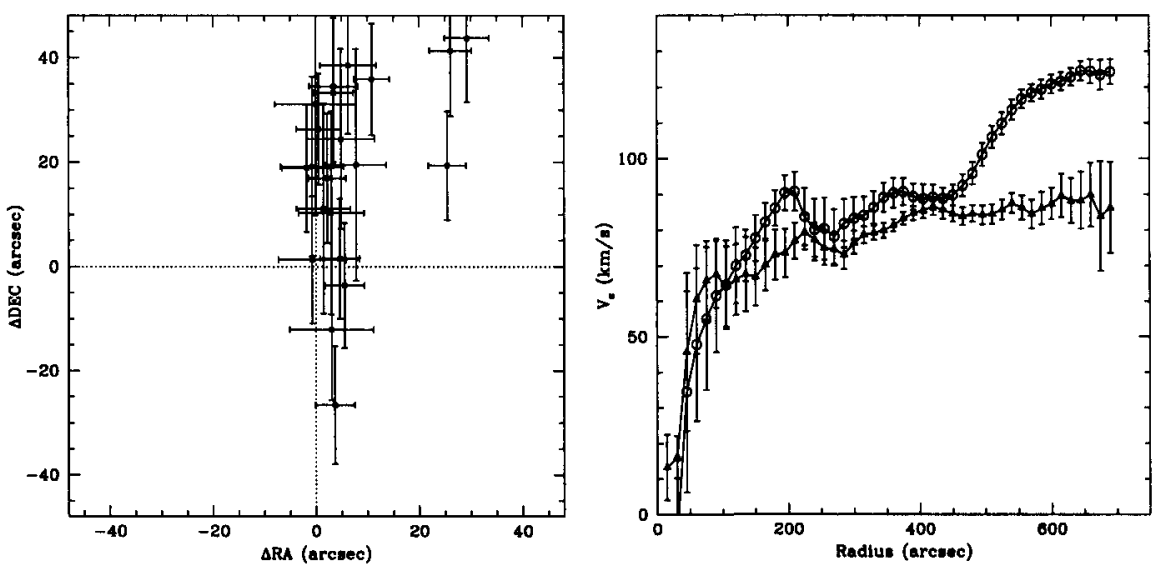

Figure 1. Left: Position of the kinematic centre of NGC 247 of all the tilted rings, relative to the optical centre; Right: Rotation curves of the approaching and receding sides separately for NGC 247. All tiltedring parameters, except of course the rotation velocity, were kept fixed in making this fit.

then starts to rise. Next, a tilted-ring fit was made for both sides simultaneously and subsequently the harmonic decomposition of the velocity field along the individual tilted-rings was made. The rotation curve is strongly influenced by the Northern Feature (a region of perturbed gas motion and a region where an arm-like extention is visible in the optical image), since it is the average of the rotation curves of the approaching and receding sides separately. The position angle and inclination are relatively constant as a function of radius, and therefore NGC 247 does not possess a significant warp. At radii corresponding to the Northern Feature, the systemic velocity drops by more than $12 \mathrm{~km} \mathrm{~s}^{-1}$. The rise in the rotation curve and the drop in systemic velocity are clearly connected to each other, as one also expects in lopsided galaxies (Swaters et al. 1999). This means that the mass decomposition of NGC 247 as presented in Carignan \& Puche (1990) is not correct, since it is based on the average rotation curve, including the rise due to the Northern feature. Instead, one would probably want to perform the mass decomposition using the rotation curve of the receding side only. On the other hand, the errorbars on their average rotation velocity are taken to include the rotation velocity of the approaching and receding sides, giving a wide range in possible mass decompositions.

Of the higher harmonics, the $\hat{s}_{3}$ term shows significant radial variation. Translated into a measurement of the elongation of the potential $\epsilon_{\text {pot }} \sin 2 \varphi_{2}$, Figure 2, left panel, we see that this quantity is relatively high in the inner 300 arcsec. This may be related to a bar-like feature seen in the optical image. After 300 arcsec the elongation shows an oscillation around zero, possibly indicating a weak two-armed spiral structure. The measurement of the lopsidedness parameter, $\lambda(R)$, is presented in Figure 2, right panel. What is immediately striking 

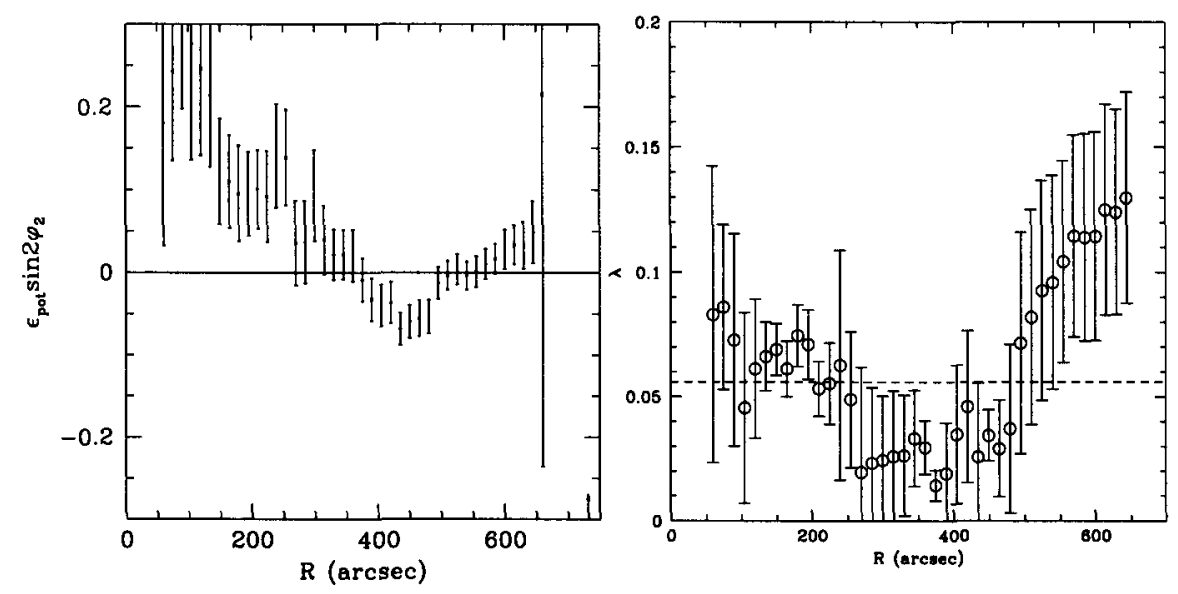

Figure 2. Left: The elongation $\epsilon_{\text {pot }} \sin 2 \varphi_{2}$ of the potential as a function of radius in NGC 247. The high value in the inner parts may be due to the bar-like feature in the centre of NGC 247; Right: The lopsidedness parameter $\lambda$ as a function of radius in NGC 247. The strong increase after 450 arcsec is caused by the Northern Feature.

is the strong increase of $\lambda$ after 450 arcsec; of course again associated with the Northern Feature. Also, one can see that there is some weak lopsidedness in the inner regions between 100 and 250 arcsec. It is not immediately obvious what might be responsible for this inner lopsidedness. It may be that the central bar is slightly offset.

In summary, NGC 247 shows a clear deviation from circular motion at radii larger than 450 arcsec, related to the Northern Feature seen in the optical image. The $\mathrm{HI}$ is elongated as well, giving the galaxy a lopsided appearance. There is no sign of warping of the plane of the $\mathrm{H}$ I disk. The Northern Feature is most likely the result of some external perturbation - the influence of a nearby dwarf galaxy or the result of an interaction with one of the other members of the Sculptor Group in the past or the infall of a dwarf galaxy - since the current velocity configuration is not stable.

\subsection{The rest of the Group and Discussion}

In table 1 , we summarize the magnitude and significance of the systematic perturbations found in the Scultor Group. While warps are a well-documented and common kinematic perturbation which is often seen in field galaxies, the strong lopsidedness and elongation measured in these systems is particularly striking. Previous analysis of more isolated galaxy samples (SFdZ, SSSvA, Schoenmakers) suggests that such strong perturbations are not particularly common. In particular, it is striking that NGC 7793 and NGC 300 show extremely high elongation measurements. Also, these two are the only galaxies with an obvious kinematic warp. In isolated galaxies these two phenomena did not seem to be coupled. 


\begin{tabular}{|l|l|l|l|l|l|l|}
\hline Galaxy & warp & $\sigma_{w}$ & $\lambda$ & $\sigma_{\lambda}$ & $\epsilon_{*}$ & $\sigma_{\epsilon}$ \\
\hline NGC 247 & 0 & 1 & 0.055 & 0.003 & 0.000 & 0.008 \\
NGC 253 & 2 & 2 & 0.049 & 0.006 & -0.037 & 0.003 \\
NGC 300 & 30 & 5 & 0.036 & 0.002 & 0.095 & 0.016 \\
NGC 55 & 4 & 3 & 0.056 & 0.008 & -0.04 & 0.003 \\
NGC 7793 & 10 & 4 & 0.019 & 0.002 & -0.130 & 0.013 \\
\hline
\end{tabular}

Table 1. The kinematic perturbations for the individual galaxies in our sample. The elongation $\epsilon_{\text {pot }} \sin \left(2 \phi_{\text {obs }}\right)$ is denoted by $\epsilon_{*}$

Also, these two galaxies have relatively low rotation velocities. There may be a weak correlation between maximum rotation velocity and the magnitude of the elongation, as Bosma pointed out during the conference. But it seems more likely that these high values are caused by the group environment: perturbation by dwarf galaxies and perturbations from past interactions amongst the five major group members themselves. If damping times for certain types of perturbations are long (especially lopsidedness and elongation), then most of the global phenomena could be due to past interactions. Walker, Mihos \& Hernquist (1996) showed that accretion of a small companion by a large disk galaxy can create perturbations that last for about a Gyr. Weinberg (1994) has shown that an $m=1$ distortion in a King model is only weakly damped. In a halo dominated galaxy, the passage of another galaxy or accretion of a satellite galaxy can thus excite an $m=1$ perturbation that can last for 10 to 100 crossing times. Distortions such as the Northern Feature in NGC 247, however, are unlikely to be the result of interactions in the distant past, since the velocity field as it is today presents us with what seems to be a highly unstable configuration. In this case, perhaps the very nearby dwarf DDO 6 is the direct cause of this phenomenon. But accretion of extra-galactic gas or a small galaxy seems equally plausible.

As far as we are aware, no extensive numerical simulations have yet been performed that simulate the internal dynamics of galaxies in a small group, although Athanassoula (priv. comm.) has performed a simulation with 5 identical galaxies in a group. It would be very interesting to quantify the type and strength of the perturbations which arise from interactions within the group environment and with different dark matter parameters. This may teach us much about the persistence of such perturbations, the way they are created and even something about the way in which dark matter halos interact and merge.

The fact that all five are kinematically lopsided to some degree, may indicate that lopsidedness is a perturbation that, once triggered in a galaxy, is quite stable and only decays slowly. This could of course also be verified in numerical experiments.

As for the high elongation measurements, it is not clear how to correctly interpret them. In principle, the interpretation of the $\hat{s}_{1}$ and $\hat{s}_{3}$ terms as indicators of elliptical orbits is based on the assumption of a stable, non-rotating, time-independent perturbation, like a triaxial dark matter halo. In the case of a fairly compact galaxy group like Sculptor, these assumptions may be inappropriate (due to interactions). On the other hand, whether or not we are allowed 
to interpret the harmonic terms as elongation, what is clear is that these kinematic harmonic terms are large and relatively constant as a function of radius in three of the five studied systems. Substantial insight into halo and galaxy kinematics would likely follow from modeling these observational results with numerical simulations.

\section{Acknowledgements}

I would like to thank Robert Braun, who has provided much of the insights and data presented here. The author would also like to thank the NCSA Astronomy Digital Image Library (ADIL) for providing images of NGC 253 and NGC 300 for this article.

\section{References}

Begeman K., 1987, $\mathrm{PhD}$-thesis, University of Groningen

Braun, R., 1995, A\&AS 114, 409

Braun, R., 1997, ApJ484, 637

Carignan, C., Puche, D., 1990, AJ, 100,641

Carignan, C., Puche, D., 1990, AJ, 100, 394

Puche, D., Carignan, C., 1988, AJ, 95, 1025

Puche, D., Carignan, C., 1991, ApJ, 378, 487

Puche, D., Carignan, C., Bosma, A., 1990, AJ, 100, 1468

Puche, D., Carignan, C., van Gorkom,J. H., 1991, AJ, 101, 456

Puche, D., Carignan, C., Wainscoat, R. J., 1991, AJ, 101, 447

Schoenmakers R. H. M., Franx M., de Zeeuw P. T., 1997, MNRAS, 292, 349

Schoenmakers R. H. M., 1999, PhD-thesis, University of Groningen

Swaters R. A., Schoenmakers R. H. M., Sancisi R., van Albada T. S., 1999, MNRAS, 304, 330

Walker I. R., Mihos C., Hernquist L., 1996, ApJ, 460, 121

Weinberg, M. D., 1994, ApJ, 421, 481 